\title{
Supervised neuromuscular exercise prior to hip and knee replacement: 12-month clinical effect and cost-utility analysis alongside a randomised controlled trial
}

\author{
Linda Fernandes ${ }^{1,2^{*}}$, Ewa M. Roos ${ }^{3}$, Søren Overgaard ${ }^{1,4}$, Allan Villadsen ${ }^{1}$ and Rikke Søgaard 5,6
}

\begin{abstract}
Background: There are indications of beneficial short-term effect of pre-operative exercise in reducing pain and improving activity of daily living after total hip replacement (THR) and total knee replacement (TKR) surgery. Though, information from studies conducting longer follow-ups and economic evaluations of exercise prior to THR and TKR is needed. The aim of the study was to analyse 12-month clinical effect and cost-utility of supervised neuromuscular exercise prior to THR and TKR surgery.
\end{abstract}

Methods: The study was conducted alongside a randomised controlled trial including 165 patients scheduled for standard THR or TKR at a hospital located in a rural area of Denmark. The patients were randomised to replacement surgery with or without an 8-week preoperative supervised neuromuscular exercise program (Clinical Trials registration no.: NCT01003756). Clinical effect was measured with Hip disability and Osteoarthritis Outcome Score (HOOS) and Knee injury and Osteoarthritis Outcome Score (KOOS). Quality adjusted life years (QALYS) were based on EQ-5D-3L and Danish preference weights. Resource use was extracted from national registries and valued using standard tariffs (2012-EUR). Incremental net benefit was analysed to estimate the probability for the intervention being cost effective for a range of threshold values. A health care sector perspective was applied.

Results: HOOS/KOOS quality of life [8.25 ( $95 \% \mathrm{Cl}, 0.42$ to 16.10)] and QALYs [0.04 (95\% Cl, 0.01 to 0.07)] were statistically significantly improved. Effect-sizes ranged between 0.09-0.59 for HOOS/KOOS subscales. Despite including an intervention cost of $€ 326$ per patient, there was no difference in total cost between groups [€132 (95\% Cl -3942 to 3679)]. At a threshold of $€ 40,000$, preoperative exercise was found to be cost effective at $84 \%$ probability.

Conclusion: Preoperative supervised neuromuscular exercise for 8 weeks was found to be cost-effective in patients scheduled for THR and TKR surgery at conventional thresholds for willingness to pay. One-year clinical effects were small to moderate and favoured the intervention group, but only statistically significant for quality of life measures.

Trial registration: ClinicalTrials.gov (NCT01003756) October 28, 2009.

Keywords: Osteoarthritis, Exercise, Cost-benefit analysis, Arthroplasty, Replacement

\footnotetext{
* Correspondence: linda.fernandes1@gmail.com

'Department of Orthopaedic Surgery and Traumatology, Odense University Hospital, Odense, Denmark

${ }^{2}$ Department of Rehabilitation, Odense University Hospital, Sdr. Boulevard 29,

5000 Odense C, Denmark

Full list of author information is available at the end of the article
} 


\section{Background}

Total hip and knee replacement (THR and TKR) surgery are recognized treatments for pain relief in patients with severe symptoms from hip or knee osteoarthritis (OA) [1]. Nevertheless, one year after surgery up to $50 \%$ of patients undergoing THR and TKR may not experience clinically important improvements in pain and activities of daily living (ADL) [2, 3]. Supervised exercise has shown to be effective treatment for reducing pain and improving ADL in patients with OA [4-9]. It seems exercise at later stages of the disease, and prior to joint replacement surgery, also has beneficial results $[10,11]$.

However, before a new treatment strategy such as preoperative exercise is implemented, one key input into the decision-making process is the effect and cost-effectiveness of the strategy in question. Today, information on the post-operative effect of exercise prior to surgery is sparse and sufficiently powered studies with feasible interventions and longer follow-ups along with high-quality economic evaluations are warranted [11-15].

We previously conducted a randomised controlled trial (RCT) evaluating an 8-week supervised neuromuscular exercise prior to THR and TKR $[10,16]$. The study showed overall improvements in favour of the exercise group in ADL prior to surgery and at 6 weeks postoperatively. At 3 months postoperatively the effects were diminished [16]. Although demonstrating short-term effects only, the addition of preoperative exercise may be clinically important in early mobilisation and returning to prior activities. Our aim with this study was to evaluate one-year clinical effect and cost-utility of the supervised neuromuscular exercise programme prior to THR and TKR. If supervised exercise prior to THR and TKR is shown to be cost-effective, health policy decision makers should consider changing the pre-operative care trajectory to include supervised exercise prior to THR and TKR.

\section{Methods}

\section{Overview of study design and participants}

165 patients were included between 4 January 2010 and 21 March 2011.[10, 16] Inclusion criteria were; $\geq 18$ years of age and scheduled for THR or TKR due to symptomatic OA. Exclusion criteria were; scheduled for bilateral surgery, previous fractures in or adjacent to the joint, inflammatory arthritis and severe heart disease or neurologic deficits. Included patients were randomly allocated to the intervention group, i.e. supervised neuromuscular exercise and preoperative educational package (EP); or to the control group, i.e. EP alone (Fig. 1). The primary outcome was the ADL subscale of the Hip disability and Osteoarthritis Outcome Score (HOOS) and Knee injury and Osteoarthritis Outcome Score (KOOS) [17-19].
Clinical effect was measured with HOOS and KOOS at one year post-surgery. Assessments points were at baseline, 8 weeks (post-intervention), 15 weeks (6 weeks postsurgery), 21 weeks (3 months post-surgery) and 61 weeks (one year post-surgery). The economic evaluation was conducted alongside the RCT and applied the health care sector perspective, incorporating cost of health care services, including cost of the exercise program. It took form of a costutility analysis using Quality Adjusted Life Years (QALYs). The time horizon was 61 weeks within the start and end date of the study (4 January 2010 - 13 August 2012).

\section{Intervention}

The neuromuscular exercise programme was supervised by a physiotherapist and focused on lower extremity muscular control and quality of movement.[10, 16, 20, 21] It consisted of three parts: warm-up, circuit programme and cool-down. The majority of the exercises were weightbearing exercises imitating functions of daily living and the patients learned how to control hip-knee-foot alignment in each exercise. Progression of exercise level was guided by neuromuscular control and quality of the performance (determined by the physiotherapist) and with acceptable exertion (determined by the patient). The programme was delivered in groups of 6-12 patients twice weekly lasting $1 \mathrm{~h}$ per session at the Department of Rehabilitation at Odense University Hospital, Svendborg, in a rural part of Southern Denmark. An attendance of 12 sessions or more was considered good compliance.

The EP was standard preoperative information on the operating procedure, expected postoperative progression and a leaflet on various exercises [16]. All patients were offered the EP.

\section{Intervention cost}

Valuation of formal care of the exercise program was based on tariff-based costs for physiotherapy in primary care (https://fysio.dk/globalassets/documents/raadgivn ing/overenskomster/praksisoverenskomster/takster-forfysioterapi-oktober-2016.pdf). The fees reflect the physiotherapist's wage, capital cost and expenses, e.g. rental costs, use of equipment, dispensable material and electricity. Implementation cost of the exercise program was not included. Costs for the 3 -h patient education package were not included as this was offered to all participants in the trial. All monetary units were reported in 2012-EUR with an exchange rate of DKK 7.45 to 1 EUR.

\section{Health care utilisation and cost}

Individual data was extracted from two national registers: The National Health Insurance Service Registry and The Danish National Patient Register [22, 23]. The former includes details about all services provided in primary care including national reimbursement fees [22]. 


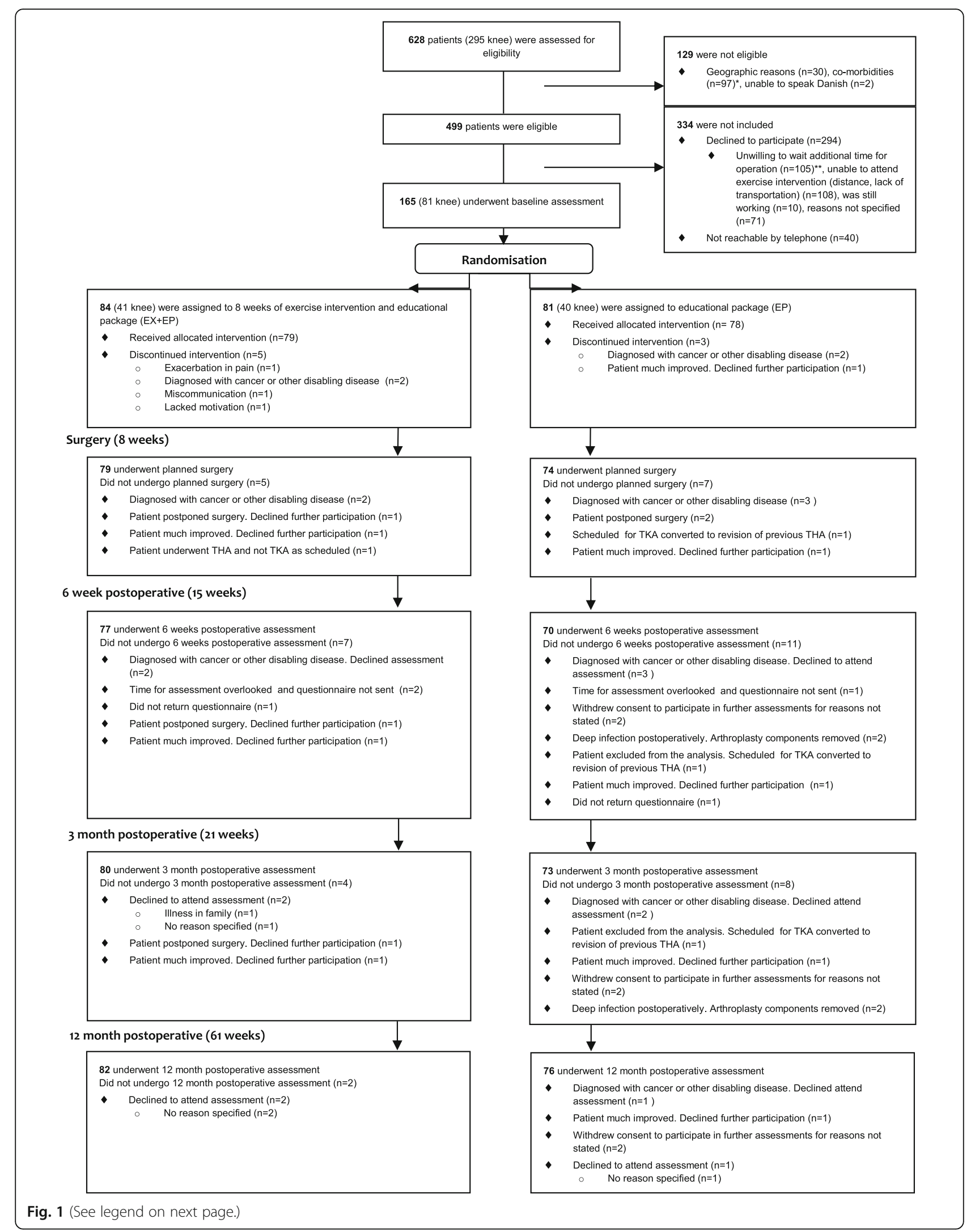


(See figure on previous page.)

Fig. 1 Flow diagram of patients participating in this study. ${ }^{*}$ Co-morbidities $(n=97)$. Previous fracture in or adjacent to the joint $(n=13)(1 \mathrm{knee})$.

- Inflammatory arthritis $(n=11)$ (5 knee). Revision arthroplasty $(n=7)$ (4 knee). Previously enrolled with another joint $(n=9)(7$ knee).

- Unicompartemental replacement (knee) $(n=27)$. Bilateral procedure in same session or within 3 month $(n=16)$. Necrosis of the femoral head (hip) $(n=6)$. Neurological disorders $(n=6)$, Hemiparesis $(n=2)$, Parkinsons Disorder $(n=2)$, Dementia $(n=2)$. Dysplasia of the femoral head $(n=1)$. Possible cancer metastasis in proximal femur $(n=1)$.** The Danish Healthcare System has a one month treatment guarantee. Entering this study meant all patients accepting an additional wait of up to 5 weeks in comparison to the treatment guarantee. After randomization, this additional wait applied only for patients randomized to the 8 week exercise intervention. The control group was operated on when originally scheduled

The latter includes details about all contacts to hospitals including diagnoses, procedures and diagnosis-relatedgrouping casemix tariffs [23].

\section{Patient expenses}

Valuation of patients' time and transport for attending the exercise classes were included in a sensitivity analysis. Valuation of informal time (i.e. time spent by patients attending the exercise regimen) was based on a human capital approach, for which the value of a person's time is reflected by wage rates (productivity loss). The wage rate was estimated by applying age- and gender matched national average gross income for year 2012 extracted from Statistics Denmark [24]. Informal time for one exercise session was set to a fixed value of $1.25 \mathrm{~h}$. Patients' expenses for travelling to and from the gym were calculated by using the national fees for travel reimbursement for 2012 (DKK $3.80 / \mathrm{km}$ or $€ 0.51 / \mathrm{km}$ ) times the distance $(\mathrm{km})$ between the exercise facility and patients' homes.

\section{Patient reported outcome measures}

The HOOS and KOOS assesses pain, symptoms, ADL, function in sport and recreation and knee related quality of life in five separate subscales scored on a 0-100 (worst to best) scale $[18,19,25,26]$.

Utility was expressed as QALYs measured with the generic outcome measure European Quality of Life 5Dimension 3-Level Health Outcome (EQ-5D-3L) [27]. Health state valuations from the Danish general population were adopted [28].

\section{Analysis}

Baseline subject characteristics were summarised as number (\%) or mean (SD). QALYs were produced by calculating the area under the curve of the EQ-5D-3L utility scores from baseline and all follow-ups assuming linear trend between observations. Visits and costing for primary care were categorised based on care provider. In hospital stay and costing for secondary care were categorised based on primary unit. All parameters were tested for normality and distribution. Because of skewed data all comparative analyses, including the net benefit, were based on bootstrapped standard errors. Non- parametric bootstrapping with 10,000 replications was applied [29].

Group comparisons were based on intention-to-treat analysis.[30] Since no interaction (group allocation joint involved) was seen in the original RCT,[16] the analyses did not adjust for hip or knee involvement. One-year clinical effect was expressed as the betweengroup mean difference [95\% confidence interval (CI)] of change values (61 weeks - baseline) and effect-size ( $d$ = mean difference of change values/pooled baseline standard deviation) of the five subscale scores of the HOOS and KOOS. Analysis of linear regression was used for between-group comparisons of QALYs and costs and presented as between-group mean differences (95\% CI) over the time horizon. An adjustment for baseline health utility was included in the analysis to account for baseline imbalances in the estimation of mean differential QALYs [31].

Handling of missing EQ-5D-3L utility scores was based on comparison of complete item response and two different imputation methods: last observation carried forward (LOCF), in which missing values are imputed based on existing values, and linear trend at point (LTAP), in which missing values are imputed by values based on a linear regression model using nonmissing observations in the series to fit the regression. Analysis comparing responders and non-responders was performed for QALYs. Since imputed data using the LTAP method showed the lowest mean difference estimate for QALYs (Table 2), and thereby the lowest risk of overestimating results, it was decided to be used in the analyses. A significance level of 0.05 was used.

\section{Cost-utility}

The cost-utility analysis adopted a health care sector perspective. We estimated the value for money of the intervention by calculating the incremental net monetary benefit using a range of hypothetical threshold values for decision-makers' willingness-to-pay for a unit of effect [32]. The threshold values ranged from $€ 0$ to $€ 100,000$. The net benefits were presented visually in cost-effectiveness acceptability curves (CEAC). These curves illustrate the probability that the intervention is cost-effective compared to the control at various 
threshold values of willingness-to-pay for a QALY gain. A willingness-to-pay of $€ 40,000$ per QALY gained was used as the threshold indicating good value for money [33, 34].

\section{Sensitivity analyses}

Four sensitivity analyses were performed to assess robustness of results for cost-utility and presented in the CEAC.

1. Complete item response analysis leaving out patients not filling in the EQ-5D-3L one or more times during the follow-up period.

2. Per-protocol analysis including only patients who complied to exercise.

3. Not adjusting analysis for the potentially skewed baseline EQ-5D-3 L.

4. Including patients' travel and time costs associated with attending exercise.

\section{Results}

Overall, 92.1\% of the observations of the HOOS or KOOS and the EQ-5D-3L at the five assessment points were complete. There were $122(74 \%)$ complete item responses for QALYs. There was no significant difference between number of patients with missing QALY in the intervention $(n=21)$ and control group $(n=22)$ $(p=0.75)$. Cost data had no missing values. Except for the EQ-5D-3L, there were no differences at baseline. Baseline characteristics are presented in Table 1. Five patients did not go through THR or TKR surgery during the 61 weeks. Reasons for declining surgery were: intervention group, much improved after exercise $(n=1)$ and no reason specified $(n=1)$; and control group, cancer $(n=1)$, started to exercise on her own $(n=1)$ and anxious about surgical procedure $(n=1)$.

\section{Patient reported outcomes}

The intervention was associated with a statistically significant QALY gain of 0.04 (95\% CI, 0.01 to 0.07 ). The QALYs showed similar results for complete item response, different imputation methods and unadjusted analyses (Table 2). Mean differences at 61 weeks favoured the exercise group for all HOOS/KOOS subscales, however only significantly so for the quality of life subscale [mean difference 8.25 points ( $95 \% \mathrm{CI}, 0.42$ to 16.10)] (Table 2). Effect-sizes for HOOS/KOOS subscales ranged from $0.09-0.59$ (Table 2).

\section{Resource use}

All patients in both groups attended the preoperative education package prior to surgery. In total, 144 exercise sessions were provided during the intervention period with a mean of 7.7 patients per session. On average,
Table 1 Baseline subject characteristics

\begin{tabular}{llll}
\hline & $\begin{array}{l}\text { Intervention } \\
\text { group }\end{array}$ & $\begin{array}{l}\text { Control } \\
\text { group }\end{array}$ & $\begin{array}{l}\text { Mean difference } \\
(95 \% \mathrm{Cl})\end{array}$ \\
\hline $\mathrm{n}$ & 84 & 81 & \\
Female sex & $47(56 \%)$ & $45(56 \%)$ & \\
Age-years & $67.9(8.6)$ & $66.9(8.3)$ & $1.08(-1.52$ to 3.67$)$ \\
BMI-kg/m ${ }^{2}$ & $29.6(4.5)$ & $31.1(6.1)$ & $-1.41(-3.05$ to 0.24$)$ \\
Waiting list THA & $43(51 \%)$ & $41(51 \%)$ & \\
Waiting list TKA & $41(49 \%)$ & $40(49 \%)$ & \\
EQ-5D-3L & $0.63(0.15)$ & $0.57(0.19)$ & $0.06(0.01$ to 0.12$)$ \\
HOOS/KOOS & & & \\
$\quad$ ADL & $50.5(15.1)$ & $45.6(16.9)$ & $4.9(-0.02$ to 9.83$)$ \\
$\quad$ Pain & $46.8(14.3)$ & $42.7(14.4)$ & $4.0(-0.39$ to 8.46$)$ \\
$\quad$ Symptoms & $49.4(19.7)$ & $44.6(18.6)$ & $4.8(-0.98$ to 10.55$)$ \\
$\quad$ Sport \& Recreation & $24.6(17.2)$ & $19.9(18.2)$ & $4.6(-0.80$ to 10.09) \\
$\quad$ Quality of life & $31.2(12.1)$ & $28.9(15.9)$ & $2.3(-2.01$ to 6.60)
\end{tabular}

Continuous variables are expressed as the mean (SD); non-continuous variables are expressed as the number of patients (\%); THA, Total hip arthroplasy; TKA, Total knee arthroplasty; EQ5D-3L, European Quality of Life 5 Dimension 3 Level Health Outcome; HOOS, Hip disability and Osteoarthritis Outcome Score; KOOS, Knee injury and Osteoarthritis Outcome Score; $A D L$, function in daily living

patients in the intervention group had attended the exercise programme 13.1 times (Table 3). Sixty-two of the 84 patients in the intervention group (74\%) displayed good compliance.

The average number of health care visits in primary and secondary care, including the number of inhospital days was not significantly different between groups, with the exception of visits with a chiropractor (Table 3). In total, 4 and 36 visits with chiropractor were registered in the intervention and control groups, respectively. $95 \%$ of the visits in the subgroup "other" had visited the dentist. None of the participants had visited psychologist funded by the national health care system during the follow-up period. Summarizing all inhospital days, $75 \%$ were to orthopaedic units.

\section{Cost}

Participating in the supervised neuromuscular exercise program cost on average (SE) €326 (12.9) per patient (Table 4). A mean of 7.7 patients attended each session. Hence, the tariff to the physiotherapist was based on groups of eight equivalent to a cost of $€ 186 /$ session (https://fysio.dk/praksis/Overenskomst-og-takster/Almenfysioterapi1/Almen-fysioterapi/). No differences between groups (€-132; 95\% CI -3668 to 3405$)$ were found for costs in primary or secondary health care sector (Table 4). The largest cost was, as expected, found for inpatient hospital stay. 
Table 2 Mean differences of QALYs and HOOS or KOOS for the 61-week follow-up period

\begin{tabular}{|c|c|c|c|c|c|c|}
\hline & \multicolumn{2}{|c|}{ Intervention $(n=84)$} & \multicolumn{2}{|c|}{ Control $(n=81)$} & \multirow[b]{2}{*}{ Mean difference $(95 \% \mathrm{Cl})$} & \multirow[b]{2}{*}{ ES } \\
\hline & $n$ & Mean (SE) & $n$ & Mean (SE) & & \\
\hline QALY, LTAP & 84 & $0.66(0.04)$ & 81 & $0.61(0.04)$ & 0.04 (0.01 to 0.07 ) & \\
\hline QALY, LTAP & 84 & $0.80(0.01)$ & 81 & $0.74(0.01)$ & 0.06 (0.02 to 0.09$)$ & \\
\hline QALY, LOCF ${ }^{a}$ & 82 & $0.64(0.05)$ & 79 & $0.58(0.05)$ & 0.05 (0.02 to 0.09$)$ & \\
\hline QALY, complete ${ }^{a}$ & 63 & $0.68(0.05)$ & 59 & $0.63(0.05)$ & 0.05 (0.02 to 0.09$)$ & \\
\hline QALY, per-protocol ${ }^{a}$ & 62 & $0.63(0.04)$ & 81 & $0.59(0.05)$ & 0.04 (0.01 to 0.07 ) & \\
\hline \multicolumn{7}{|l|}{$\mathrm{HOOS} / \mathrm{KOOS}$} \\
\hline $\mathrm{ADL}$ & 84 & $35.9(2.1)$ & 81 & $32.1(2.4)$ & $3.80(-2.45$ to 10.07$)$ & 0.24 \\
\hline Pain & 84 & $41.2(2.2)$ & 81 & $37.1(2.4)$ & $4.13(-2.33$ to 10.60$)$ & 0.29 \\
\hline Symptoms & 84 & $33.9(2.7)$ & 81 & $32.2(2.6)$ & $1.69(-5.79$ to 9.17$)$ & 0.09 \\
\hline Sport and Recreation & 84 & $33.1(3.6)$ & 81 & $26.3(2.8)$ & $6.79(-2.10$ to 15.69$)$ & 0.39 \\
\hline Quality of Life & 84 & $43.4(2.6)$ & 81 & $35.2(3.0)$ & 8.25 (0.42 to 16.10$)$ & 0.59 \\
\hline
\end{tabular}

Mean (bootstrap SE) and mean differences (95\% confidence interval). ES, effect-size (mean difference/pooled standard deviation)

$Q A L Y$, quality-adjusted life-years; complete, complete item response analysis; LTAP, linear trend at point; $L C O F$, last observation carried forward; per-protocol,

per-protocol analysis equals attending $\geq 12$ exercise sessions. HOOS, Hip disability and Osteoarthritis Outcome Score; KOOS, Knee injury and Osteoarthritis Outcome Score; $A D L$, function in daily living

${ }^{a}$ Adjusted for baseline EQ-5D-3 L scores

bUnadjusted analysis

Patients attending exercise travelled on average $21.5 \mathrm{~km}$ (range $0.5-78.3 \mathrm{~km}$ ) to the exercise facility and had a mean (SE) transportation cost of $€ 137$ (12.2). The mean (SE) informal time valuation was €302 (15.3) for the intervention period. When including patients' expenses, the cost for the intervention increased to a mean (SE) of $€ 765$ (33.5) per patient during the intervention period.

\section{Cost-utility}

At conventional thresholds, decision-makers willingness to pay around $€ 40,000$ the probability for the intervention being cost-utile was estimated at $84 \%$. Sensitivity analyses showed that the cost-utility result was robust (Fig. 2).

\section{Discussion}

The present analysis demonstrates that a pre-operative 8 -week supervised exercise intervention was costeffective, showing a probability of $84 \%$ for decisionmakers willingness to pay around $€ 40,000$ for a QALY gained. This is comparable to the typical threshold between $£ 20,000$ and $£ 30,000$ per QALY gained used by the British National Health Service when instituting new treatments.[34] Compared to care as usual, we found no overall additional health care cost during the first postoperative year despite adding 8 weeks of physiotherapistsupervised exercise prior to TKR and THR surgery. In return one can expect QALY gain over the following year.

This study is one of few RCTs that estimates costs and cost-effectiveness of exercise as treatment in patients with hip and knee OA, and, to our knowledge, the first to analyse cost-utility of exercise prior to THR and TKR. Previous cost-effectiveness analyses evaluating exercise, as the only intervention and not prior to surgery, in patients with knee OA found better health outcomes at lower costs, i.e. exercise was cost saving [13]. One RCT found that water-based exercise saved $£ 123-175$ per patient per year despite a relatively high intervention cost (£830 per patient) [35]. A second RCT found that both aerobic and resistance training were cost saving, \$114 and $\$ 117$, respectively, along with improvements in selfreported functioning [36]. A third RCT found QALY gains of 0.023 (SE 0.04) from class-based exercise compared to home-based exercise and a probability of $70 \%$ of being cost-effective at a willingness to pay of $£ 30.000$ [37]. Further, two RCTs have shown less total costs for patients with hip OA and chronic knee pain, respectively, following patient education and exercise programs compared to usual care [38, 39]. Comparing our study of exercise prior to OA surgery to studies evaluating exercise interventions in other patient groups, the results are quite similar. A Cochrane review of exercise for patients with heart failure reported a QALY gain of 0.03 and a probability of $90 \%$ for willingness to pay around 50.000 USD [40], group-based exercise for the prevention of falling showed an incremental cost per QALY gain of 72.700 AUD [41], and pelvicfloor muscle training showed cost-effectiveness with a probability of $>70 \%$ for the willingness to pay around $£ 50.000[42]$.

We found that patients allocated to exercise had a lower total length of hospital stay and total cost during the follow-up period. The results were not statistically significant. However, should these findings not be due to 
Table 3 Health care utilization during 61 weeks

\begin{tabular}{|c|c|c|c|}
\hline & $\begin{array}{l}\text { Intervention } \\
\text { group }(n=84)\end{array}$ & $\begin{array}{l}\text { Control group } \\
(n=81)\end{array}$ & \\
\hline & & & $\begin{array}{l}\text { Mean difference } \\
(95 \% \mathrm{Cl})\end{array}$ \\
\hline Intervention & & & \\
\hline $\begin{array}{l}\text { Exercise sessions, } \\
\text { mean (range) }\end{array}$ & $13.1(0-24)$ & 0 & $13.1(12.2$ to 14.0$)$ \\
\hline Primary health care, vis & & & \\
\hline General practice & $18.2(1.3)$ & $19.5(1.7)$ & $-1.36(-5.46$ to 2.74$)$ \\
\hline Physiotherapist & $0.3(0.1)$ & $0.5(0.2)$ & $-0.27(-0.81$ to 0.27$)$ \\
\hline Medical specialist & $0.9(0.2)$ & $1.0(0.2)$ & $-0.05(-0.57$ to 0.48$)$ \\
\hline Chiropractor & 0 & $0.4(0.03)$ & $-0.40(-0.77$ to -0.03$)$ \\
\hline Other ${ }^{a}$ & $2.0(0.1)$ & $2.2(0.2)$ & $-0.28(-0.72$ to 0.16$)$ \\
\hline Subtotal & $21.4(1.3)$ & $23.7(1.7)$ & $-2.36(-6.61$ to 1.90$)$ \\
\hline Secondary health care, & jisits & & \\
\hline Outpatient & $8.4(0.9)$ & $8.5(1.0)$ & $-0.13(-2.80$ to 2.55$)$ \\
\hline Emergency & $0.2(0.1)$ & $0.5(0.1)$ & $-0.24(-0.52$ to 0.03$)$ \\
\hline Subtotal & $8.6(0.9)$ & $9.0(1.1)$ & $-0.37(-3.16$ to 2.43$)$ \\
\hline Secondary health care, & nhospital days & & \\
\hline Orthopeadic & $3.6(0.4)$ & $4.6(1.1)$ & $-0.95(-3.32$ to 1.42$)$ \\
\hline Surgery ${ }^{\mathrm{b}}$ & $0.3(0.1)$ & $1.2(0.7)$ & $-0.91(-2.28$ to 0.45$)$ \\
\hline Medicine $^{c}$ & $0.7(0.3)$ & $0.8(0.3)$ & $-0.12(-0.92$ to 0.68$)$ \\
\hline Oncology ${ }^{d}$ & 0 & $0.4(0.3)$ & $-0.37(-0.95$ to 0.22$)$ \\
\hline Other & $0.3(0.2)$ & $0.3(0.3)$ & $-0.01(-0.72$ to 0.70$)$ \\
\hline Subtotal & $4.8(0.7)$ & $7.2(1.8)$ & $-2.36(-6.11$ to 1.38$)$ \\
\hline
\end{tabular}

Variables are expressed as the mean (bootstrap SE) number of outpatient visits or in-hospital days per patient during the 61 week follow-up period and the mean difference ( $95 \%$ confidence interval) between groups

${ }^{\mathrm{b}}$ Surgery, gastrointestinal, urology, plastic, thoracic

'Medicine, internal medicine, cardiology, medical gastroenterology, neurology, geriatrics, general practice

${ }^{d}$ Other, includes inhospital stay at oftamology, odontology or
${ }^{a}$ Other, includes visits at the dentistry, laboratory or foot care clinic physiotherapy units

Table 4 Costs of the intervention, outpatient and emergency visits and inpatient hospital stay during 61 weeks

\begin{tabular}{|c|c|c|c|}
\hline & $\begin{array}{l}\text { Intervention } \\
\text { group }(n=84)\end{array}$ & $\begin{array}{l}\text { Control group } \\
(n=81)\end{array}$ & \\
\hline & & & $\begin{array}{l}\text { Mean difference } \\
(95 \% \mathrm{Cl})\end{array}$ \\
\hline Intervention & & & \\
\hline Physiotherapy & $326(13)$ & 0 & 326 (301 to 351 ) \\
\hline Primary health care se & & & \\
\hline General Practice & $331(28)$ & $345(29)$ & $-14(-92$ to 64$)$ \\
\hline Physiotherapist & $22(10)$ & $98(61)$ & $-76(-197$ to 45$)$ \\
\hline Specialists & $99(21)$ & $97(29)$ & $2(-68$ to 72$)$ \\
\hline Chiropractor & $2(1)$ & $6(2)$ & $-4(-9$ to 0$)$ \\
\hline Other $^{\mathrm{a}}$ & $75(7)$ & $83(8)$ & $-7(-28$ to 14$)$ \\
\hline Subtotal & $530(35)$ & $629(74)$ & -99 ( -258 to 60$)$ \\
\hline Secondary health care & sector & & \\
\hline Outpatient & $2240(560)$ & $1917(317)$ & 323 (-933 to 1579$)$ \\
\hline Emergency & $12(4)$ & $32(11)$ & $-20(-43$ to 4$)$ \\
\hline Subtotal & $2252(557)$ & $1949(316)$ & 303 (-949 to 1555$)$ \\
\hline Secondary health care & sector & & \\
\hline Orthopaedics & $11760(572)$ & $11695(1038)$ & 66 (-2250 to 2382$)$ \\
\hline Surgery ${ }^{\mathrm{b}}$ & $280(180)$ & $773(363)$ & $-493(-1285$ to 300$)$ \\
\hline Medicine ${ }^{c}$ & $883(322)$ & $865(350)$ & 18 (-919 to 955$)$ \\
\hline Oncology & 0 & $213(170)$ & -213 ( -547 to 120$)$ \\
\hline Other $^{d}$ & $150(149)$ & $189(169)$ & $-40(-494$ to 415$)$ \\
\hline Subtotal & 13074 (706) & 13735 (1249) & $-662(-3478$ to 2154$)$ \\
\hline TOTAL & 16181 (1174) & $16313(1374)$ & $-132(-3668$ to 3405$)$ \\
\hline
\end{tabular}

Variables are expressed as the mean (bootstrap SE) per patient during the 61 week follow-up period and the mean difference (95\% confidence interval) between groups. The monetary units are presented in EUR2012

${ }^{a}$ Other, includes visits at the dentistry, laboratory or foot care clinic

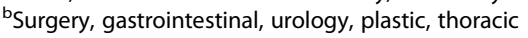

'Medicine, internal medicine, cardiology, medical gastroenterology, neurology, geriatrics, general practice

${ }^{\mathrm{d}}$ Other, includes inhospital stay at oftamology, odontology or physiotherapy units

random variation, a reduction of 2.4 days in hospital per patient and year is a relevant difference for the health care system. In 2012, a total of 8787 and 8008 patients underwent THR and TKR, respectively, at Danish hospitals [43, 44]. Extrapolating the resource use to the Danish THR and TKR population in 2012, exercise prior to surgery could potentially save 39.500 days in hospital per year. Seventy-five percent of all in hospital days during the 61 week period were at orthopaedic units, leaving $25 \%$ at other units. We did not ask the patients about comorbidities, but the data show that at least some had concurrent diseases. The second largest cost, after admission to orthopaedic units, was admission to internal medicine and cardiology units (Table 4). Over half of the population with hip and knee OA have been found to have concomitant cardiovascular disease and $86 \%$ of patients going through THR or TKR have one or more comorbidities $[45,46]$. There have also been found significant associations between number and type of comorbidity and lower ADL, pain and HRQoL scores, with largest impact on ADL in THR patients, suggesting that functional limitations due to other diseases have to be taken into account to optimize outcome after THR and TKR $[45,46]$. A national database study from Taiwan found a reverse dose-relationship of having coronary artery disease and dyslipidemia in patients attending physiotherapy due to symptoms from their OA, i.e. patients receiving a higher dose of physiotherapy showed a lowered risk of coronary artery disease and dyslipidemia [47]. Since comorbidities are common in OA and possibly can be influenced by the intervention given in our study, we find the inclusion of the total cost and resource use important for this study (Table 3 and 4).

The sensitivity analyses showed that the health care sector perspective using LTAP imputed data was robust 


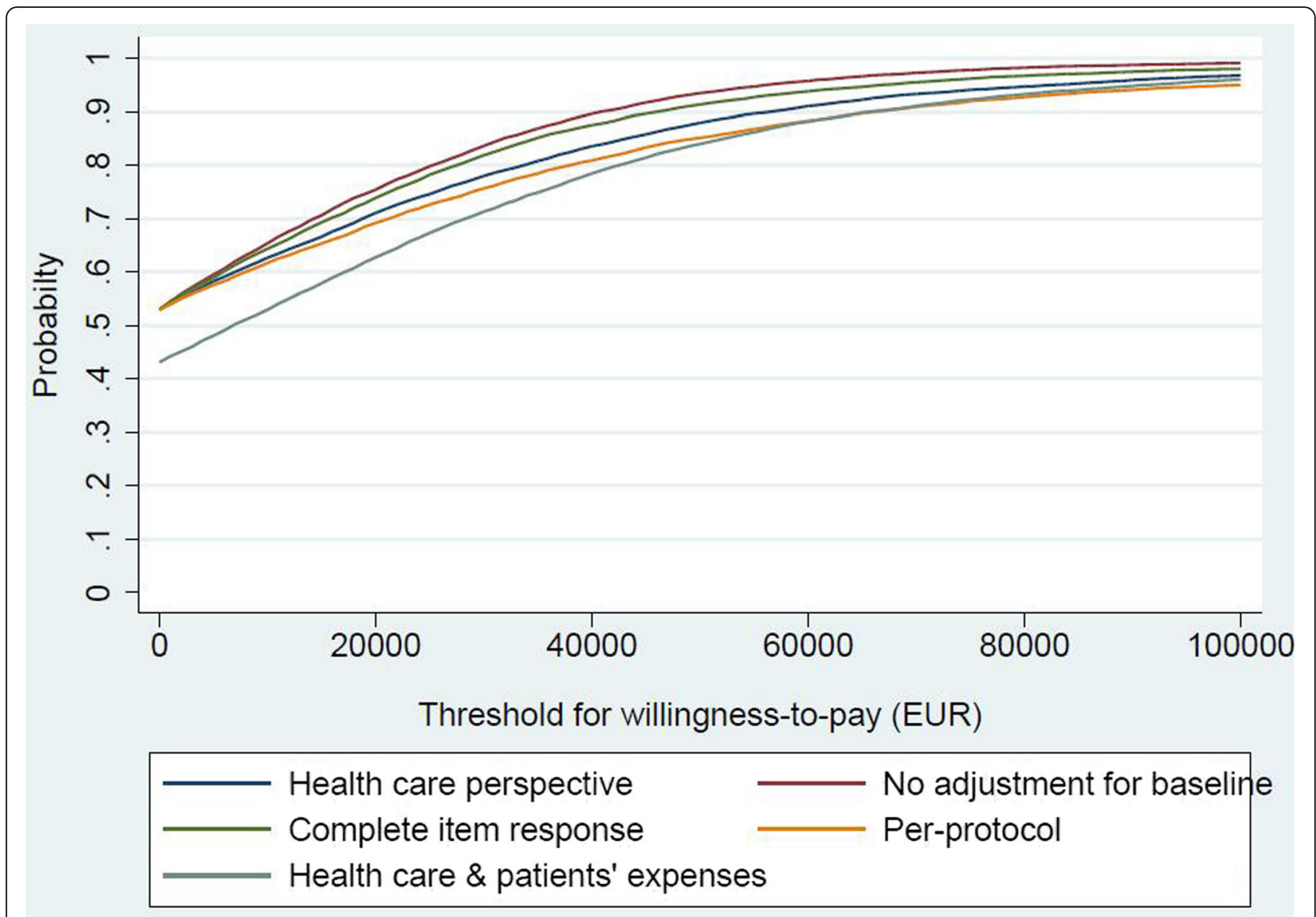

Fig. 2 Cost effectiveness acceptability curves for incremental net monetary benefit to estimate the probability for the intervention being cost effective at conventional thresholds for willingness to pay. Health care perspective, Health Care Sector perspective (base-case analysis); Complete item response, only complete item response of the EQ-5D-3L included in the analysis; Health care \& patients' expenses, Health Care Sector and patients' own expenses perspective; No adjustment for baseline, adjustments for baseline EQ-5D-3L scores were not included; Per-protocol, only patients attending 12 or more exercise sessions were included in the analysis

as compared to complete item response, unadjusted and per-protocol analyses. The analysis using the health care sector plus patients' expenses differed slightly from the base-case analysis showing a slightly lower probability for willingness to pay thresholds. This was expected since adding patients' average expenses by $€ 439$ to the average cost of the intervention $(€ 326)$ would result in higher costs for the intervention group, i.e. $€ 765$ versus $€ 326$ for the intervention per patient calculated with or without patient expenses, respectively. This study had a follow-up period of 61 week. We did not find it necessary to discount for costs or consequences as the study only passed one year by 9 weeks. Implementation cost of the intervention was not included in the analysis as the intervention today is available at 377 private and public physiotherapy clinics nationwide in Denmark [48]. Hence, the exercise program is already on the market in Denmark and could be implemented as standard care for patients undergoing THR or TKR without extra costs for education and training of care providers.
With regards to generalisability of the cost-utility results there are challenges in interpretation due to the vast differences in health care structure worldwide. In Denmark, all health care utilization and cost for different services and procedures performed in primary and secondary care are registered in national registers [22, 23]. To enable comparison to other health care structures, the overall average unit costs in this study can be found by dividing the cost estimates in table 4 with the resource use in table 3 or by viewing the tariff catalogues [49]. However, the efficacy of the intervention is well documented and it is of general interest to optimise the pre- as well as postoperative period with increased activities of daily living and through this a possible reduction in postoperative complications (e.g. joint stiffness, thrombosis/emboli) and a faster return to work for the younger part of this patient group.

\section{Strength and limitations}

Some strengths of this RCT were a rigorous design by applying the CONSORT recommendations [30], evaluating 
a time horizon of one year, in which changes in ADL and HRQoL are expected to appear [50, 51], and using a common generic HRQoL measurement (EQ-5D-3L) to calculate QALYs [52]. Our sample size of 165 allowed us however to detect moderate, as opposed to small, effect sizes. We found a significant effect size of 0.59 favouring the exercise group in HOOS/KOOS quality of life, but the effect size of 0.39 in HOOS/KOOS sport and recreation function remained non-significant (Table 2). One limitation of this RCT was that the Danish national registers do not include costs for care delivered directly by the municipality. In Denmark, the municipality is responsible for post-operative care after hospital discharge, e.g. standard post-operative exercise and home-care. Although group allocation was stratified on municipality, differences in resource use and costs between the groups during the follow-up period may exist. Another limitation was that we had no data on patients' work status (sick-leave, disability pension, retired or in the workforce) or OA-related medicine. The majority of participants in this study were assumed to be retired, as the retirement age in Denmark is 65 years and the average age in the study was 67.5 years at baseline. Even though the majority was assumed to be retired, a recent study has shown that the year after THR and TKR patients cost $€ 6000$ more compared to a reference population due to loss of employment income, use of medication and need for home care [53]. A broader societal perspective including also work status, medication and municipality-based services would therefore have been optimal. If this intervention would be implemented in routine clinical practice, the intervention could be provided in the primary care setting usually located within $15 \mathrm{~km}$ from peoples' homes. The private pocket cost of transportation would then be reduced whereas program administration costs might increase and gains from economies of scale could be lost. This should be considered and balanced with the potential benefit of extra participation and/or compliance by patients sensitive to provider and/or transportation distance. Finally, of those meeting eligibility criteria for this study only $30 \%$ were included which may impact external validity.

\section{Conclusion}

Preoperative supervised neuromuscular exercise for 8 weeks was found to be cost-effective in patients scheduled for THR and TKR surgery at conventional thresholds for willingness to pay. One-year clinical effects were small to moderate and favoured the intervention group, but only statistically significantly so for quality of life measures.

\section{Abbreviations}

ADL: Activities of Daily Living; CEAC: Cost-Effectiveness Acceptability Curves; Cl: Confidence Interval; CONSORT: Consolidated Standards of Reporting Trials; DKK: Danish Krona; EQ-5D-3L: European Quality of Life 5-Dimension 3-Level
Health Outcome; HOOS: Hip disability and Osteoarthritis Outcome Score; HRQoL: Health Related Quality of Life; KOOS: Knee injury and Osteoarthritis Outcome Score; LOCF: Last Observation Carried Forward; LTAP: Linear Trend At Point; OA: Osteoarthritis; QALY: Quality Adjusted Life Year;

RCT: Randomised Controlled Trial; SD: Standard Deviation; SE: Standard Error; THR: Total Hip Replacement; TKR: Total Knee Replacement; USD: US Dollar

\section{Funding}

This study was financed by research grants from OUH Svendborg Hospital (nr. 12/9062) and the Association of Danish Physiotherapists' Foundation for research, CPD and professional development. The funders had no role in the study design, data collection and analysis, decision to publish or preparation of the manuscript.

\section{Availability of data and materials}

All data from the study are presented in the manuscript. We encourage the sharing of data. Please contact the corresponding author if you wish to gain access to data presented in this study.

\section{Authors contribution}

All authors were responsible for concept and design. LF and AV were responsible of collection of data. LF was responsible for assembly of data and drafting of the article. LF and RS were responsible for the analysis of data. RS was responsible for statistical expertise. LF, RS, ER, AV and SO were responsible for interpretation of data, critical revision of the article for important intellectual content and for final approval of the article. LF and RS take responsibility for the integrity of the work as a whole from inception to finished article.

\section{Competing interests}

$L F$ is co-owner of Ther-ex Ltd, Denmark. AV is co-owner of Ther-ex Ltd., Denmark. Ther-ex Ltd distribute (free of charge and commercial free) the mobile application Ther-ex which offers functional and neuromuscular exercise, exercise diary and pain monitoring for people with hip and knee osteoarthritis. ER is developer of the Good Life with Osteoarthritis in Denmark (GLA:D) program. GLA:D is a not-for-profit initiative to implement clinical guidelines for osteoarthritis, hosted at the University of the Southern Denmark. All other authors declare no competing interests.

\section{Consent for publication}

Not applicable.

\section{Ethics and consent to participate}

Informed written consent was obtained and the study was approved by The Committee for Biomedical Research Ethics for the Region of Southern Denmark, identifier: S-20090099 and the Danish Data Protection Agency, identifier: 13/6464. The trial is registered at ClinicalTrials.gov (NCT01003756).

\section{Author details}

${ }^{1}$ Department of Orthopaedic Surgery and Traumatology, Odense University Hospital, Odense, Denmark. ${ }^{2}$ Department of Rehabilitation, Odense University Hospital, Sdr. Boulevard 29, 5000 Odense C, Denmark. ${ }^{3}$ Research Unit for Musculoskeletal Function and Physiotherapy, Institute of Sports Science and Clinical Biomechanics, University of Southern Denmark, Odense, Denmark. ${ }^{4}$ Department of Clinical Research, University of Southern Denmark, Odense, Denmark. ${ }^{5}$ Department of Public Health, Aarhus University, Aarhus, Denmark. ${ }^{6}$ Department of Clinical Medicine, Aarhus University, Aarhus, Denmark.

Received: 8 June 2016 Accepted: 19 December 2016

Published online: 06 January 2017

\section{References}

1. Felson DT, Lawrence RC, Hochberg MC, McAlindon T, Dieppe PA, Minor MA, Blair SN, Berman BM, Fries JF, Weinberger M, et al. Osteoarthritis: New insights. Part 2: treatment approaches. Ann Intern Med. 2000;133(9):726-37.

2. Hawker GA, Badley EM, Borkhoff CM, Croxford R, Davis AM, Dunn S, Gignac MA, Jaglal SB, Kreder HJ, Sale JE. Which patients are most likely to benefit from total joint arthroplasty? Arthritis Rheum. 2013;65(5):1243-52.

3. Paulsen $A$, Roos EM, Pedersen AB, Overgaard S. Minimal clinically important improvement (MCII) and patient-acceptable symptom state (PASS) in total 
hip arthroplasty (THA) patients 1 year postoperatively. Acta Orthop. 2014;85(1):39-48.

4. Fransen M, McConnell S. Exercise for osteoarthritis of the knee. Cochrane Database Syst Rev. 2008;4:CD004376.

5. Fransen M, McConnell S, Hernandez-Molina G, Reichenbach S. Exercise for osteoarthritis of the hip. Cochrane Database Syst Rev. 2009;3:CD007912.

6. Lange AK, Vanwanseele B, Fiatarone Singh MA. Strength training for treatment of osteoarthritis of the knee: a systematic review. Arthritis Care Res. 2008:59(10):1488-94.

7. Fernandes L, Hagen KB, Bijlsma JW, Andreassen O, Christensen $P$, Conaghan PG, Doherty M, Geenen R, Hammond A, Kjeken I, et al. EULAR recommendations for the non-pharmacological core management of hip and knee osteoarthritis. Ann Rheum Dis. 2013;72(7):1125-35.

8. Hernandez-Molina G, Reichenbach S, Bin Z, Lavalley M, Felson DT. Effect of therapeutic exercise for hip osteoarthritis pain: results of a meta-analysis. Arthritis Care Res. 2008:59(9):1221-8

9. Juhl C, Christensen R, Roos EM, Zhang W, Lund H. Impact of exercise type and dose on pain and disability in knee osteoarthritis: a systematic review and meta-regression analysis of randomized controlled trials. Arthritis Rheumatol. 2014;66(3):622-36.

10. Villadsen A, Overgaard S, Holsgaard-Larsen A, Christensen R, Roos EM. Immediate efficacy of neuromuscular exercise in patients with severe osteoarthritis of the hip or knee: a secondary analysis from a randomized controlled trial. J Rheumatol. 2014;41(7):1385-94.

11. Wallis JA, Taylor NF. Pre-operative interventions (non-surgical and nonpharmacological) for patients with hip or knee osteoarthritis awaiting joint replacement surgery - a systematic review and meta-analysis. Osteoarthritis Cartilage. 2011;19(12):1381-95.

12. Hoogeboom TJ, Oosting E, Vriezekolk JE, Veenhof C, Siemonsma PC, de Bie RA, van den Ende CHM, van Meeteren NLU. Therapeutic validity and effectiveness of preoperative exercise on functional recovery after joint replacement: a systematic review and meta-analysis. PLoS ONE. 2012;7(5):e38031.

13. Pinto $D$, Robertson MC, Hansen P, Abbott JH. Cost-effectiveness of Nonpharmacologic, nonsurgical interventions for Hip and/or knee osteoarthritis: systematic review. Value Health. 2012;15(1):1-12.

14. Puig-Junoy J, Ruiz Zamora A. Socio-economic costs of osteoarthritis: a systematic review of cost-of-illness studies. Semin Arthritis Rheum. 2015;44(5):531-41.

15. Kwok IH, Paton B, Haddad FS. Does Pre-operative physiotherapy improve outcomes in primary total knee arthroplasty? - a systematic review. J Arthroplasty. 2015;30(9):1657-63.

16. Villadsen A, Overgaard S, Holsgaard-Larsen A, Christensen R, Roos EM. Postoperative effects of neuromuscular exercise prior to hip or knee arthroplasty: a randomised controlled trial. Ann Rheum Dis. 2014;73(6): 1130-7.

17. Roos EM, Roos HP, Lohmander LS, Ekdahl C, Beynnon BD. Knee injury and osteoarthritis outcome score (KOOS)-development of a self-administered outcome measure. J Orthop Sports Phys Ther. 1998;28(2):88-96.

18. Roos EM, Toksvig-Larsen S. Knee injury and osteoarthritis outcome score (KOOS) - validation and comparison to the WOMAC in total knee replacement. Health Qual Life Outcomes. 2003;1:17.

19. Nilsdotter AK, Lohmander LS, Klassbo M, Roos EM. Hip disability and osteoarthritis outcome score (HOOS)-validity and responsiveness in total hip replacement. BMC Musculoskelet Disord. 2003;4:10.

20. Ageberg $E$, Link A, Roos EM. Feasibility of neuromuscular training in patients with severe hip or knee OA: the individualized goal-based NEMEX-TJR training program. BMC Musculoskelet Disord. 2010;11:126.

21. Ageberg E, Nilsdotter A, Kosek E, Roos EM. Effects of neuromuscular training (NEMEX-TJR) on patient-reported outcomes and physical function in severe primary hip or knee osteoarthritis: a controlled before-and-after study. BMC Musculoskelet Disord. 2013;14:232.

22. Sahl Andersen J, De Fine ON, Krasnik A. The Danish national health service register. Scand J Public Health. 2011;39(7 suppl):34-7.

23. Lynge E, Sandegaard JL, Rebolj M. The Danish national patient register. Scand J Public Health. 2011:39(7 suppl):30-3.

24. Gross and Disposable income by region, gender and age range, Table INDKP5. http://www.statistikbanken.dk/statbank5a/default.asp?w=1920.

25. Roos EM, Lohmander LS. The knee injury and osteoarthritis outcome score (KOOS): from joint injury to osteoarthritis. Health Qual Life Outcomes. 2003;1:64.
26. Peer MA, Lane J. The knee injury and osteoarthritis outcome score (KOOS): a review of its psychometric properties in people undergoing total knee arthroplasty. J Orthop Sports Phys Ther. 2013;43(1):20-8.

27. EuroQol Group; Oemar MO, M.: EQ-5D-3 L User Guide. Basic information on how to use the EQ-5D-3 L instrument. . In. http://www.eurogol.org/; 2013.

28. Wittrup-Jensen KU, Lauridsen J, Gudex C, Pedersen KM. Generation of a Danish TTO value set for EQ-5D health states. Scand J Public Health. 2009;37(5):459-66.

29. Efron BT, Tibshirani R. Bootstrap methods for standard errors, confidence intervals and other measures of statistical accuracy. Stat Sci. 1986;1 (1):54-77.

30. Schulz KF, Altman DG, Moher D, Group C. CONSORT 2010 statement: updated guidelines for reporting parallel group randomised trials. BMJ. 2010;340:C332.

31. Manca A, Hawkins N, Sculpher MJ. Estimating mean QALYs in trial-based cost-effectiveness analysis: the importance of controlling for baseline utility. Health Econ. 2005;14(5):487-96.

32. Hernández MAN, Vázquez-Polo FJ, González-Torre FJG, Bas EM. Complementing the net benefit approach: a new framework for Bayesian cost-effectiveness analysis. Int J Technol Assess Health Care. 2009;25(04):537-45.

33. Eichler H-G, Kong SX, Gerth WC, Mavros P, Jönsson B. Use of costeffectiveness analysis in health-care resource allocation decision-making: How Are cost-effectiveness thresholds expected to emerge? Value Health. 2004; $7(5): 518-28$.

34. The National Institute for Health and Care Excellence. How NICE measures value for money in relation to public health interventions. 2013. https:// www.nice.org.uk/Media/Default/guidance/LGB10-Briefing-20150126.pdf.

35. Cochrane T, Davey R, Matthes Edwards S. Randomised controlled trial of the cost-effectiveness of water-based therapy for lower limb osteoarthritis. Health Technol Assess. 2005;9(31):130.

36. Sevick MA, Bradham DD, Muender M, Chen GJ, Enarson C, Dailey M, Ettinger WH. Cost-effectiveness of aerobic and resistance exercise in seniors with knee osteoarthritis. Med Sci Sports Exerc. 2000;32(9):1534-40.

37. Richardson G, Hawkins N, McCarthy CJ, Mills PM, Pullen R, Roberts C, Silman A, Oldham JA. Cost-effectiveness of a supplementary class-based exercise program in the treatment of knee osteoarthritis. Int J Technol Assess Health Care. 2006;22(1):84-9.

38. Juhakoski R, Tenhonen S, Malmivaara A, Kiviniemi V, Anttonen T, Arokoski JP. A pragmatic randomized controlled study of the effectiveness and cost consequences of exercise therapy in hip osteoarthritis. Clin rehabil. 2011;25(4):370-83.

39. Hurley MV, Walsh NE, Mitchell H, Nicholas J, Patel A. Long-term outcomes and costs of an integrated rehabilitation program for chronic knee pain: a pragmatic, cluster randomized, controlled trial. Arthritis Care Res. 2012;64(2):238-47.

40. Rees K, Taylor RS, Singh S, Coats AJ, Ebrahim S. Exercise based rehabilitation for heart failure. Cochrane Database Syst Rev. 2004;3:CD003331.

41. Church J, Goodall S, Norman R, Haas M. An economic evaluation of community and residential aged care falls prevention strategies in NSW. N S W Public Health Bull. 2011;22(3-4):60-8.

42. Imamura M, Abrams P, Bain C, Buckley B, Cardozo L, Cody J, Cook J, Eustice S, Glazener C, Grant A, et al. Systematic review and economic modelling of the effectiveness and cost-effectiveness of non-surgical treatments for women with stress urinary incontinence. Health Technol Assess (Winch Eng). 2010;14(40):1-188. iii-iv.

43. Dansk Hoftealloplastik Register. Årsrapport 2013. . In: Dækker fra 1 januar til 31december 2012 Opgørelseperiode fra 1 januar 2012 til 31 December 2012 Edited by Overgaard SP, A.B. www.dhr.dk; 2013.

44. Dansk Knæalloplastikregister. Årsrapport 2013. Administrativt ekstrakt. . In: Kvalitetsindikatorer for operationer foretaget i perioden 2008-2012 Den fulde rapport dækker perioden 01011997 - 31122012 Edited by Pedersen A, B.; Haagen Hjelm, A.; Odgaard, A. www.dkar.dk; 2013.

45. Peter WF, Dekker J, Tilbury C, Tordoir RL, Verdegaal SHM, Onstenk R, Bénard MR, Vehmeijer SB, Fiocco M, Vermeulen HM, et al. The association between comorbidities and pain, physical function and quality of life following hip and knee arthroplasty. Rheumatol Int. 2015;1-9.

46. Kirkness CS, Yu J, Asche CV. The effect on comorbidity and pain in patients with osteoarthritis. J Pain Palliat Care Pharmacother. 2008;22(4):336-48.

47. Yeh HJ, Chou YJ, Yang NP, Cheng CC, Huang N. Physical therapy reduces coronary artery disease and dyslipidemia among osteoarthritis patients: a nationwide database study. Arch Phys Med Rehabil. 2015;97:8-16.

48. Skou ST, Odgaard A, Rasmussen JO, Roos EM. Group education and exercise is feasible in knee and hip osteoarthritis. DanMed J. 2012;59(12):A4554. 
49. Ministeriet for sundhed og forebyggelse. Takstsystem 2012. http:// sundhedsdatastyrelsen.dk/-/media/sds/filer/finansiering-og-afregning/ takster/2012/takstsystem2012.pdf. In.; 2012.

50. Vissers MM, Bussmann JB, Verhaar JAN, Arends LR, Furlan AD, Reijman M. Recovery of physical functioning after total Hip arthroplasty: systematic review and meta-analysis of the literature. Phys Ther. 2011;91(5):615-29.

51. Hopley CDJ, Crossett LS, Chen AF. Long-term clinical outcomes and survivorship after total knee arthroplasty using a rotating platform knee prosthesis: a meta-analysis. J Arthroplast. 2013;28(1):68-77. e63.

52. The National Institute for Health and Clinical Excellence. Methods for the development of NICE public health guidance (third edition). In., 3rd edn. https://www.nice.org.uk/process/pmg4/chapter/introduction; 2012.

53. Kjellberg J, Kehlet $\mathrm{H}$ : A nationwide analysis of socioeconomic outcomes after hip and knee replacement. Dan Med J 2016, 63 (8).

Submit your next manuscript to BioMed Central and we will help you at every step:

- We accept pre-submission inquiries

- Our selector tool helps you to find the most relevant journal

- We provide round the clock customer support

- Convenient online submission

- Thorough peer review

- Inclusion in PubMed and all major indexing services

- Maximum visibility for your research

Submit your manuscript at www.biomedcentral.com/submit
Biomed Central 